\title{
Effective Typestate Verification in the Presence of Aliasing
}

\author{
Stephen Fink* \\ Eran Yahav* \\ Nurit Dor ${ }^{\dagger}$ \\ G. Ramalingam* \\ Emmanuel Geay* \\ * IBM T.J. Watson Research Center \\ \{sjfink,eyahav,grama,egeay\}@us.ibm.com \\ $\dagger$ IBM Haifa Research Lab \\ nurit@il.ibm.com
}

\begin{abstract}
This paper addresses the challenge of sound typestate verification, with acceptable precision, for real-world Java programs.

We present a novel framework for verification of typestate properties, including several new techniques to precisely treat aliases without undue performance costs. In particular, we present a flowsensitive, context-sensitive, integrated verifier that utilizes a parametric abstract domain combining typestate and aliasing information. To scale to real programs without compromising precision, we present a staged verification system in which faster verifiers run as early stages which reduce the workload for later, more precise, stages.

We have evaluated our framework on a number of real Java programs, checking correct API usage for various Java standard libraries. The results show that our approach scales to hundreds of thousands of lines of code, and verifies correctness for $93 \%$ of the potential points of failure.
\end{abstract}

Categories and Subject Descriptors: D.2.4[Software Engineering]: Software/Program Verification

General Terms: Algorithms, Verification

Keywords: Typestate, Program Verification, Alias Analysis

\section{INTRODUCTION}

Statically checking if programs satisfy specified safety properties can help identify defects early in the development cycle, thus increasing productivity, reducing development costs, and improving quality and reliability.

Typestate [32] is an elegant framework for specifying a class of temporal safety properties. Typestates can encode correct usage rules for many common libraries and application programming interfaces (APIs) (e.g. [33, 2]). For example, typestate can express the property that a Java program should not read data from java.net. Socket until the socket is connected.

This paper addresses the challenge of typestate verification, with acceptable precision, for real-world Java programs.

We focus on sound verification; if the verifier reports no problem, then the program is guaranteed to satisfy the desired proper-

Permission to make digital or hard copies of all or part of this work for personal or classroom use is granted without fee provided that copies are not made or distributed for profit or commercial advantage and that copies bear this notice and the full citation on the first page. To copy otherwise, to republish, to post on servers or to redistribute to lists, requires prior specific permission and/or a fee.

ISSTA'06, July 17-20, 2006, Portland, Maine, USA.

Copyright 2006 ACM 1-59593-263-1/06/0007 ...\$5.00. ties. However, if the verifier reports potential problems, they may or may not indicate actual program errors. Imprecise analysis can lead a verifier to produce "false positives": reported problems that do not indicate an actual error. Users will quickly reject a verifier that produces too many false positives.

While the most sophisticated and precise analyses can reduce false positives, such analyses typically do not scale to real programs. Real programs typically rely on large and complex supporting libraries, which the analyzer must process in order to reason about program behavior.

This paper presents several new typestate verification techniques, ranging from the simple but imprecise, to the fairly precise but somewhat expensive. We also present a staged typestate verification approach, which exploits verifiers with varying cost/precision trade-offs. Early stages employ the efficient but imprecise analyses; subsequent stages employ progressively more expensive and precise techniques. Each progressively more precise stage focuses on verifying only "parts" of the program that previous stages failed to verify.

The key technical challenge facing typestate verification for Java concerns pointer aliasing. Since all structured data in Java is heapallocated, almost all interesting operations involve pointer dereferencing. Further, Java libraries encourage layers of encapsulation around data, which leads to multiple levels of pointer dereferencing. In order to prove that a program manipulates an object correctly, the verifier must cut through the tangle of alias relationships by which the program manipulates the object of interest.

Researchers have developed a variety of efficient flow-insensitive may-alias (pointer) analysis techniques (e.g. [10, 21]). Unfortunately, may-alias analysis is inadequate for most typestate verification problems, which require strong updates [6]. To support strong updates and more precise alias analysis, we present a framework to check typestate properties by solving a flow-sensitive, contextsensitive dataflow problem on a combined domain of typestate and pointer information. As is well-known [9], a combined domain allows a more precise solution than could be obtained by solving each domain separately. Furthermore, the combined domain allows the framework to concentrate computational effort on alias analysis only where it matters to the typestate property. This concentration allows more precise alias analysis than would be practical if applied to the whole program.

\subsection{Contributions}

The main contributions of this paper are:

- a flow-sensitive, context-sensitive, integrated verifier that utilizes a parametric abstract domain that combines typestate and points-to abstractions.

- two new techniques to handle destructive updates, utilizing 


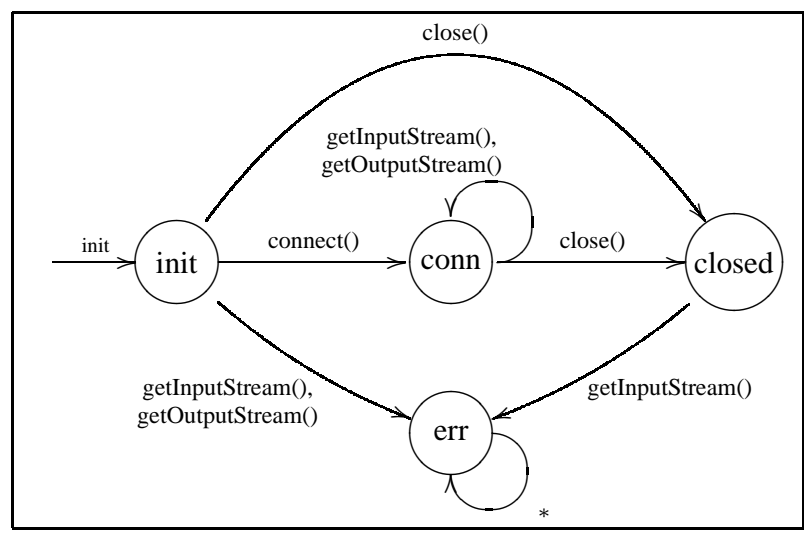

Figure 1: Partial typestate specification for java.net. Socket.

information from a preceding flow-insensitive may points-to analysis. Specifically,

- a uniqueness analysis that can strengthen the results of the may points-to analysis to support "strong updates" under certain conditions, and

- a focus operation, similar in spirit to the one used in shape analysis [31], that enables the analysis to use strong updates in certain cases.

Though inspired by shape analysis techniques, our focus operation applies to a more efficient, abstract domain, and results in analyses that are orders of magnitude more scalable than typical shape analyses.

- an empirical evaluation of the efficiency and precision of various verification techniques. The empirical results shed light on the relative importance of various techniques for treating aliases, and demonstrate the validity of a staged approach.

Our implementation handles the full Java language, excluding concurrency, subject to caveats described regarding dynamic language features such as reflection. The experimental results show that the staged solver verifies correctness for $93 \%$ of the potential points of failure, running in under 10 minutes across a suite of moderately-sized programs.

The rest of this paper is organized as follows: Sec. 2 provides an informal overview of the various challenges in typestate verification, and sketches our solutions. Sec. 3, Sec. 4 and Sec. 5 present the abstractions and techniques formally. Sec. 6 presents the empirical evaluation, and Sec. 7 reviews related work.

\section{OVERVIEW}

\subsection{Typestate Verification}

A typestate property can be specified using a finite state automaton. States in the automaton correspond to typestates which an object can occupy during execution. The automaton also contains a designated typestate err corresponding to an erroneous state of the object. Transitions in the automaton correspond to observable operations that may change the object's typestate. In this paper, we focus on observable operations corresponding to method invocations. The goal of typestate checking is to statically verify that no object reaches its error typestate during any program execution.

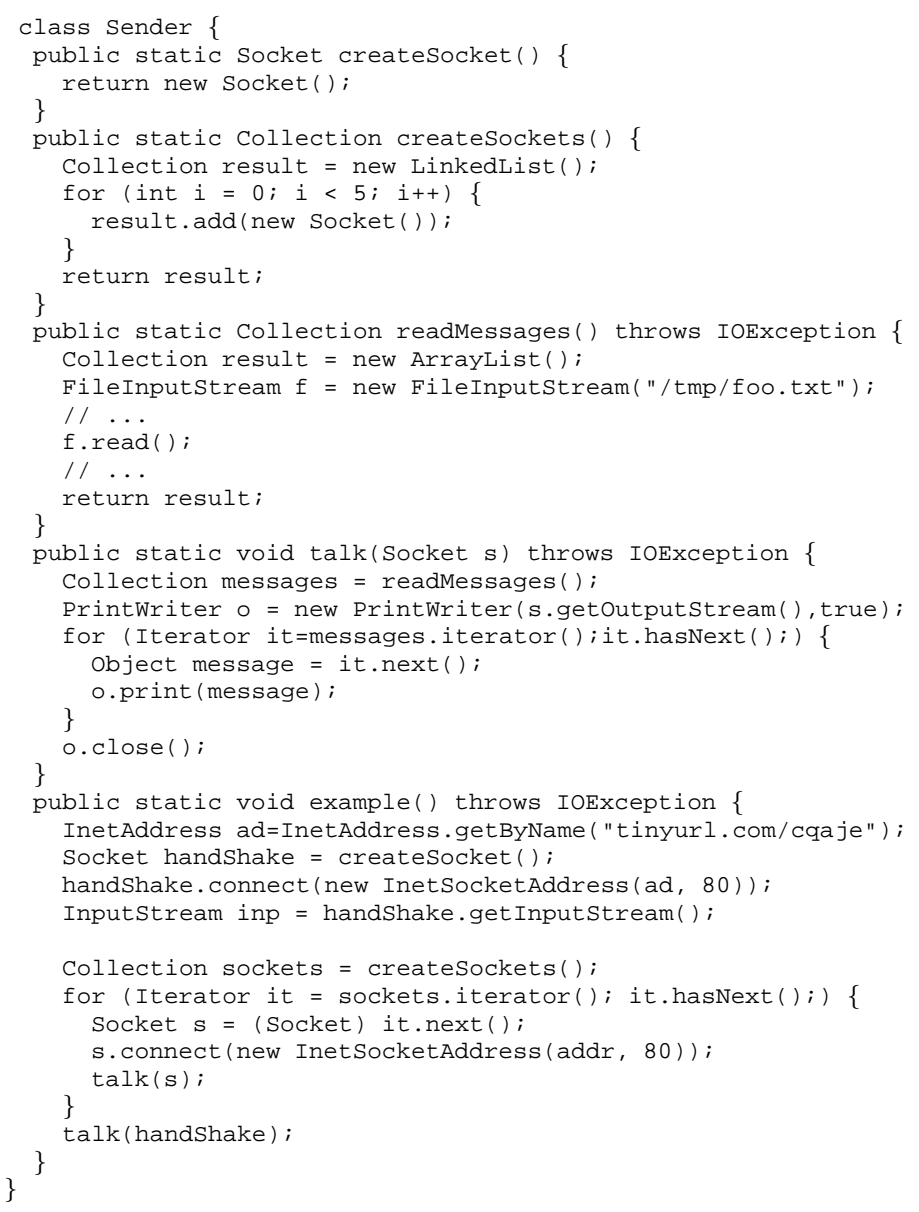

Figure 2: Program with correct usages of common APIs.

Fig. 1 shows a finite state automaton providing a partial specification for the java.net. Socket API. This automaton shows, for example, that calling get Input Stream () is only legal after a preceding call to connect () .

Fig. 2 presents a program that exercises Java Sockets, I/O streams, and Iterators. Our goal is to verify that the program

- never calls get InputStream () or get Output Stream () on a Socket unless it is connected,

- never calls read () on a closed stream, and

- always calls hasNext () on an Iterator before calling next ().

In the example program, some typestate properties (e.g.Iterators) could be verified relatively easily by local, intra-procedural reasoning. Unfortunately, any local alias analysis can be easily defeated by unknown side effects from procedure calls.

Other properties require more powerful (and costly) techniques. In particular, socket usage in the example requires an interprocedural analysis with relatively precise alias analysis, since the socket objects flow across procedure boundaries and through complex collection data structures.

\subsection{Outline of our Algorithm}

Our verification system is a composite verifier built out of several composable verifiers of increasing precision and cost. Each verifier 


\begin{tabular}{|c|c|c|c|c|c|}
\hline $\begin{array}{l}\text { Initial } \\
\text { verification } \\
\text { scope }\end{array}$ & $\rightarrow \begin{array}{c}\text { Flow-ins. } \\
\text { feasibility } \\
\text { check }\end{array}$ & $\rightarrow \begin{array}{c}\text { Intra- } \\
\text { procedural } \\
\text { verifier }\end{array}$ & & $\begin{array}{l}\text { Integrated } \\
\text { verifier }\end{array}$ & $\rightarrow \begin{array}{c}\text { Possible failure } \\
\text { points }\end{array}$ \\
\hline
\end{tabular}

Figure 3: Overview of framework stages.

can run independently, but the composite verifier stages analyses in order to improve efficiency without compromising precision. The early stages use the faster verifiers to reduce the workload for later, more precise, stages.

All of our verifiers use the results of a preceding flow-insensitive, selectively context-sensitive subset-based pointer analysis. This analysis produces a conservative approximation of the heap, and induces a partition of concrete objects into abstract objects; as is typical, the pointer analysis creates names for abstract objects based on static allocation sites and the governing context-sensitivity policy ${ }^{1}$. The flow-insensitive alias analysis can be performed relatively efficiently, and scales to large programs (e.g. [21, 24]).

Given a program and a typestate property, we consider all operations in the program that may cause a transition to an error state as points of potential failure $(P P F)$. We consider a pair $(o, p)$ where $O$ is an abstract object, and $p$ a point of potential failure, as a separate verification problem. We refer to such pairs as potential failure pairs. We define a verification scope to be a set of potential failure pairs.

Our verification system starts by initializing the verification scope to contain all matching pairs of abstract typestate objects and potential points of failure. The verification scope is then gradually reduced by a sequence of stages, as shown in Fig. 3. Each stage may successfully eliminate potential failure pairs by verifying for a pair $(o, p)$ that a failure cannot occur for objects represented by $o$ at the point $p$.

Each composable verifier exploits separation $[11,35]$ : it performs the typestate checking separately for each abstract object of the appropriate type in the program. It accepts as a parameter a verification scope which holds information from the preceding stages about which potential failure pairs remain unverified.

Each verifier restricts its attention to the verification scope, and produces an updated verification scope for the subsequent phase. The system reports any potential failure pairs that remain after the last stage as potential errors.

In the following discussion we briefly describe each of these stages. Later, we present a more detailed description of the algorithms.

\subsubsection{Flow-Insensitive Feasibility Checking}

Prior to any flow-sensitive analysis, the first stage prunes the verification scope using an extremely efficient flow-insensitive errorpath feasibility check. The flow-insensitive pointer analysis provides the set of observable operations that may occur for each abstract object. The flow-insensitive verifier determines if it is possible for the abstract object to reach the err state in the typestate automaton, using this set of operations.

Any abstract object that does not exhibit a feasible error-path could be considered as verified.

In our running example, the FileInput St ream object allocated in readMessages () is pruned at this stage, as the program never invokes close () for this abstract object, and thus it can never reach an error state (for "read () after close ()").

\footnotetext{
${ }^{1}$ Sec. 6 gives details on our implementation's context-sensitivity policy.
}

This stage, however, is unable to verify the correct usage of the Iterators or Sockets in the example program.

\subsubsection{Intraprocedural Verifier}

The intraprocedural verifier is a flow-sensitive verifier that restricts the scope of each verification attempt to a single procedure. The verification starts at the beginning of each procedure assuming an arbitrary unknown initial context (state). Method calls are treated conservatively, without analyzing the method. This essentially works well for "local" objects, which are pointed-to by local variables only. The intraprocedural verifier uses the same abstraction as the integrated verifier (see Sec. 2.2.4 and Sec. 5).

When the intraprocedural verifier is able to verify all uses of an abstract object in the program, we can avoid interprocedural verification for that object. This is often the case for typestate objects that do not escape the method in which they are allocated.

For example, the intraprocedural verifier can verify that all Iterators in our running examples are used correctly. Applying the intraprocedural verifier as an early stage eliminates the need for verification of the Iterators in the running example by the latter, more expensive interprocedural solvers.

\subsubsection{Strong Updates: Uniqueness Analysis}

While a flow-insensitive alias analysis suffices to check feasibility of an error-path (as in Sec. 2.2.1), it generally does not suffice for verifying typestate properties. A flow-insensitive analysis produces only may alias information and not must alias information. Therefore, an analyzer that directly uses the results of a flowinsensitive analysis must use "weak updates" to handle assignments and operations via a pointer.

Using "weak updates" precludes verification of many typestate properties. For example, it is insufficient for verifying the typestate property of Fig. 1. Using only may alias information, the analyzer cannot guarantee that a connect ( ) operation occurs on the same concrete object as a subsequent get Input St ream () operation. Hence, such analysis cannot verify this property.

We now present a verifier that is still based on flow-insensitive alias analysis, but uses a novel uniqueness analysis to allow strong updates in some scenarios.

Consider the invocation of a method, via a pointer $p$, that may alter the typestate of the receiver object. If the following two conditions hold, then the analysis can apply a strong update to change the typestate of the receiver object:

(a) the points-to set for $p$ consists of a single abstract object

(b) this abstract object represents a single concrete object ${ }^{2}$.

Consider an abstract object $S$ representing a particular (contextsensitive) allocation site $A$. This abstract object represents all concrete objects that are allocated at $A$. The Unique solver performs a flow- and context-sensitive analysis with a simple abstraction to determine if more than one object allocated at $A$ can be simultaneously alive. If not, then the abstract object $S$ represents at most one concrete object at that program point, and the verifier can exploit strong updates at that point if condition (a) mentioned above also holds.

For example, the Unique verifier can verify the correct use of the socket pointed-to by handShake in the method example (), despite the fact that this object is used interprocedurally (and hence

\footnotetext{
${ }^{2}$ For purposes of typestate checking, we may safely ignore the possibility of the pointer $\mathrm{p}$ being null, which will result in a nullpointer dereference exception. If desired, null-pointer checking is done separately.
} 
could not be handled by the intraprocedural verifier of the previous section).

Uniqueness analysis is of general use in our framework, and later stages incorporate the technique. This novel analysis compares favorably to existing techniques for computing unique abstract locations, as it relies on flow- and context-sensitive analysis of a pruned program with respect to the tracked abstract object (see discussion in Sec. 7).

\subsubsection{Integrated Verifier}

The Integrated verifier improves upon the Unique verifier by performing flow- and context-sensitive verification with an abstraction that combines aliasing information with typestate information. The use of a combined domain is more precise than separately performing typestate checking and flow-sensitive alias analysis, as is common with abstract interpretation over combined domains [9].

For example, flow-sensitivity of alias information enables strongupdates in cases such as the one below, where the Unique verifier fails because the abstract file object does not qualify as unique.

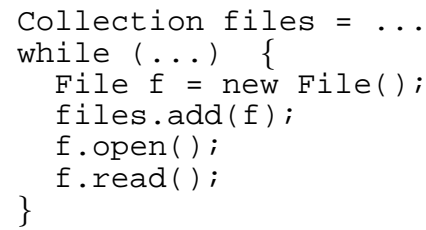

Since all our verifiers exploit separation, it suffices to focus on the problem of verifying usage for a single abstract object. The Integrated verifier utilizes an abstract domain that captures information about the typestate of the given abstract object, as well as information about a set $M$ of pointer access paths that definitely point to the given abstract object, and a set $M N$ of pointer access paths that definitely do not point to the given abstract object. The domain also includes a boolean flag indicating if there may exist other access paths, not mentioned in $M$, that may point to the given abstract object. Sec. 5 presents a more complete description of the abstraction.

A key element of the integrated verifier's abstraction is the use of a focus operation [31], which is used to dynamically (during analysis) make distinctions between objects that the underlying basic points-to analysis does not distinguish. For example, consider the loop in the method example () in our running example. The verifier utilizes two or more abstract objects to represent the set of all (5) Socket objects created by the createSockets () method (even though the flow-insensitive pointer analysis represents them by a single abstract object): one abstract object represents the Socket pointed to by $\mathrm{s}$, and the other abstract objects represent the remaining Sockets.

This enables the use of strong updates, allowing verification for all Sockets in the running example, despite their flow through a collection and across procedures.

\section{TYPESTATE CHECKING FRAMEWORK}

This section presents a framework for typestate checking which enables declaration of different levels of abstractions.

First, we sketch an instrumented concrete semantics for this problem. Intuitively, given a typestate property, our semantics instruments the program state, state $e^{\natural}$ to include for every object, $o^{\natural}$, its typestate from the property definition. The instrumented semantics verifies that an object never reaches its error typestate.

Next, we present a parameterized conservative abstraction that allows us to define the family of abstractions used by the various verifiers in our framework.

\subsection{Instrumented Concrete Semantics}

We assume a standard concrete semantics which defines a program state and evaluation of an expression in a program state. The semantic domains are defined in a standard way as follows:

$$
\begin{array}{ll}
L^{\natural} & \in \text { objects }^{\natural} \\
v^{\natural} & \in \text { Val }=\text { objects }^{\natural} \cup\{\text { null }\} \\
\rho^{\natural} & \in \text { Env }=\text { VarId } \rightarrow \text { Val } \\
h^{\natural} & \in \text { Heap }=\text { objects }{ }^{\natural} \times \text { FieldId } \rightarrow \text { Val } \\
\text { state }^{\natural}=\left\langle L^{\natural}, \rho^{\natural}, h^{\natural}\right\rangle & \in \text { States }=2^{\text {objects }^{\natural}} \times \text { Env } \times \text { Heap }
\end{array}
$$

where objects ${ }^{\natural}$ is an unbounded set of dynamically allocated objects, VarId is a set of local variable identifiers, and FieldId is a set of field identifiers.

A program state keeps track of the set of allocated objects $\left(L^{\natural}\right)$, an environment mapping local variables to values $\left(\rho^{\natural}\right)$, and a mapping from fields of allocated objects to values $\left(h^{\natural}\right)$.

We also define the notion of an access path as follows: A pointer path $\gamma \in \Gamma=$ FieldId $^{*}$ is a (possibly empty) sequence of field identifiers. The empty sequence is denoted by $\epsilon$. We use the shorthand $f^{k}$ where $f \in$ FieldId to mean a sequence of length $k$ of accesses along a field $f$. An access path $p \equiv x . \gamma \in \operatorname{VarId} \times \Gamma$ is a pair consisting of a local variable $x$ and a pointer path $\gamma$.

We denote by $A P S$ all possible access paths in a program. The 1-value of access path $p$, denote by $\operatorname{state}^{\natural}[p]$, is recursively defined using the environment and heap mappings, in the standard manner.

We formally define a typestate property as follows.

DEFINITION 3.1. A typestate property $\mathcal{F}$ is represented by a $\mathrm{fi}$ nite state automaton $\mathcal{F}=\langle\Sigma, \mathcal{Q}, \delta$, init, $\mathcal{Q} \backslash\{$ err $\}\rangle$ where $\Sigma$ is the alphabet of observable operations, $\mathcal{Q}$ is the set of states, $\delta$ is the transition function mapping a state and an operation to a successor state, init $\in \mathcal{Q}$ is a distinguished initial state, err $\in \mathcal{Q}$ is a distinguished error state for which for every $\sigma \in \Sigma, \delta($ err,$\sigma)=$ err, and all states in $\mathcal{Q} \backslash\{$ err $\}$ are accepting states. Given a sequence of operations we say that it is valid when it is accepted by $\mathcal{F}$, and invalid otherwise.

Our instrumented concrete semantics instruments every concrete state $\left\langle L^{\natural}, \rho^{\natural}, h^{\natural}\right\rangle$ with an additional mapping typestate ${ }^{\natural}: L^{\natural} \rightarrow \mathcal{Q}$ that maps an allocated object to its typestate.

For a given state state $^{\natural}=\left\langle L^{\natural}, \rho^{\natural}, h^{\natural}\right\rangle$, we define a function $A P_{\text {state }}^{\natural}: L^{\natural} \rightarrow 2^{A P s}$ as a mapping between allocated objects and the access paths that evaluate to them, i.e. $A P^{\natural}\left(o^{\natural}\right)=\{e \mid$ state $\left.^{\natural}[e]=o^{\natural}\right\}$. When the state is clear from context, we omit it and simply write $A P^{\natural}\left(o^{\natural}\right)$.

A state of the instrumented concrete semantics is therefore a tuple $\left\langle L^{\natural}, \rho^{\natural}, h^{\natural}\right.$, typestate $\left.^{\natural}\right\rangle$.

EXAMPLE 3.2. Given the property of Fig. 1, the instrumented concrete state before the first call to s. connect () in example () contains six objects: one object $o_{0}^{\natural}$ allocated during the invocation of createSocket ( ), and five other objects $o_{1}^{\natural}, \ldots, o_{5}^{\natural}$, allocated during the invocation createsockets (). The values of typestate $e^{\natural}$ and the function $A P^{\natural}\left(o_{0}^{\natural}\right)$ are:

$$
\begin{aligned}
\text { typestate }^{\natural}\left(o_{0}^{\natural}\right)=\text { conn } & A P^{\natural}\left(o_{0}^{\natural}\right)=\{\text { handShake }\} \\
\text { typestate }^{\natural}\left(o_{1}^{\natural}\right)=\text { init } & A P^{\natural}\left(o_{1}^{\natural}\right)=\{\mathrm{s}, \text { sockets.head }\} \\
\text { typestate }^{\natural}\left(o_{i}^{\natural}\right)=\text { init } & A P^{\natural}\left(o_{i}^{\natural}\right)=\left\{\text { sockets.head.next }{ }^{i-1}\right\} \\
\text { where }(i=2, . .5) &
\end{aligned}
$$

The instrumented semantics updates the typestate of the object in a natural way. When the object is first allocated, its typestate is mapped to the initial state of the typestate automaton. Then, on every observable event, the object typestate is updated accordingly. 


\subsection{Abstract Semantics}

The instrumented concrete semantics uses an unbounded set of objects with an unbounded set of (unbounded) access paths. In this section, we describe a parameterized abstract semantics that allows us to conservatively represent the instrumented concrete semantics with various degrees of precision and cost.

Our abstract semantics uses a combination of two representations to abstract heap information: (i) a global heap-graph representation encoding the results of a flow insensitive points-to analysis; (ii) enhanced flow-sensitive must points-to information integrated with typestate checking.

\subsubsection{Flow-insensitive May Points-to Information}

The first component of our abstraction is a global heap graph, obtained through a flow-insensitive, context-sensitive subset based may points-to analysis [3]. This is fairly standard and provides a partition of the set objects ${ }^{\natural}$ into abstract objects. In this discussion, we define an instance key to be an abstract object name assigned by the flow-insensitive pointer analysis. The heap graph provides for an access path $e$, the set of instance keys it may point-to and also the set of access paths that may be aliased with $e$.

The heap graph representation of the running example contains two instance keys for type Socket: one representing the object allocated in createsocket, denoted by $o_{0}^{\natural}$ in Example 3.2, and another one, for the second allocation site, representing all five objects in the sockets collection.

\subsubsection{Parameterized Typestate Abstraction}

Our parameterized abstract representation uses tuples of the form: $\left\langle\right.$ o, unique, typestate, $A P_{\text {must }}$, May, $\left.A P_{\text {mustNot }}\right\rangle$ where:

- $o$ is an instance key.

- unique indicates whether the corresponding allocation site has a single concrete live object.

- typestate is the typestate of instance key $o$.

- $A P_{\text {must }}$ is a set of access paths that must point-to $o$.

- May is true indicates that there are access paths (not in the must set) that may point to $o$.

- $A P_{\text {mustNot }}$ is a set of access paths that do not point-to $o$.

This parameterized abstract representation has four dimensions, for the length and width of each access path set (must and must-not). The length of an access path set indicates the maximal length of an access path in the set, similar to the parameter $k$ in k-limited alias analysis. The width of an access path set limits the number of access paths in this set.

An abstract state is a set of tuples. We observe that a conservative representation of the concrete program state must obey the following properties:

(a) An instance key can be indicated as unique if it represents a single object for this program state.

(b) The access path sets (the must and the must-not) do not need to be complete. This does not compromise the soundness of the staged analysis due to the indication of the existence of other possible aliases.

(c) The must and must-not access path sets can be regarded as another heap partitioning which partitions an instance key into the two sets of access paths: those that a) must alias this abstract object, and b) definitely do not alias this abstract object. If the must-alias set is non-empty, the must-alias partition represents a single concrete object.

(d) If May = false, the must access path is complete; it contains all access paths to this object.

This can be formally stated as follows:

Definition 3.3. A tuple

$\left\langle o\right.$, unique, typestate, $A P_{\text {must }}$, May, $\left.A P_{\text {must Not }}\right\rangle$ is a sound representation of object $o^{\natural}$ at instrumented state istate ${ }^{\natural}$ when:

$$
\begin{aligned}
o & =i k\left(o^{\natural}\right) \\
& \wedge \text { unique } \Rightarrow\left\{x^{\natural} \in \text { live }\left(\text { istate }^{\natural}\right) \mid i k\left(x^{\natural}\right)=o\right\}=\left\{o^{\natural}\right\} \\
& \wedge \text { typestate }=\text { typestate }^{\natural}\left(o^{\natural}\right) \wedge A P_{\text {must }} \subseteq A P^{\natural}\left(o^{\natural}\right) \\
& \wedge\left(\neg \text { May } \Rightarrow\left(A P_{\text {must }}=A P^{\natural}\left(o^{\natural}\right)\right)\right) \\
& \wedge A P_{\text {mustNot }} \cap A P^{\natural}\left(o^{\natural}\right)=\emptyset
\end{aligned}
$$

where ik is an abstraction mapping a concrete object to the instance key that represents it, and live $\left(\right.$ istate $\left.^{\natural}\right)$ is defined to be $\left\{x^{\natural} \mid A P^{\natural}\left(x^{\natural}\right) \neq \emptyset\right\}$.

DEFINITION 3.4. An abstract state istate is a sound representation of a concrete state istate ${ }^{\natural}=\left\langle L^{\natural}, \rho^{\natural}, h^{\natural}\right.$, typestate $\left.{ }^{\natural}\right\rangle$ if for every object $o^{\natural} \in L^{\natural}$ there exists a tuple in istate that provides a sound representation of $o^{\natural}$.

\subsection{Base Abstraction}

The Base (least precise) abstraction is an instance of the parameterized abstraction with zero length and width of both the must and the must-not access path sets (and hence May = true in all tuples). In addition, this abstraction does not track uniqueness. This yields a typestate checking algorithm, similar to [11] in its alias handling, that cannot verify any property that requires strong updates. For simplicity, we denote each tuple in this abstraction as $\langle o$, typestate $\rangle$

EXAMPLE 3.5. A base abstraction representing the concrete state described in Example 3.2 contains two instance keys: $o_{0}$ representing $o_{0}^{\natural}$ and $o_{1 . .5}$ representing the five objects $o_{i}^{\natural}, i=1,2, . .5$ in the socket s collection and the following three tuples: $\left\langle o_{0}\right.$, init $\rangle$, $\left\langle o_{0}\right.$, conn $\rangle,\left\langle o_{1 . .5}\right.$, init $\rangle$.

This analysis is an iterative flow- and context-sensitive propagation, that tracks tuples starting with an initial $\langle o$, init $\rangle$ generated at an allocation. The analysis only needs to handle observable operations and propagates tuples according to typestate changes. The result of an observable operation associated with event $o p$ on the tuple $\langle$ o, typestate $\rangle$ are two tuples: The previous tuple and the tuple $\langle o, \delta$ (typestate, $\mathrm{op})\rangle$. Tuples are never removed; all operations are handled as weak updates. The first tuple in Example 3.5 demonstrates the results of a weak-update. It represents that $o_{0}^{\sharp}$, in Example 3.2, may be in the init state, which is not feasible in any concrete state at this program point.

\section{UNIQUENESS ANALYSIS}

The Unique verifier extends the Base abstraction, adding an abstraction which determines whether more than one concrete object corresponding to a given instance key can be simultaneously alive. This information allows the verifier to use strong updates under certain conditions. We refer to this analysis as uniqueness analysis.

In terms of the abstraction tuples introduced in Sec. 3, the Unique verifier makes use of only the instance key, uniqueness flag, and the typestate. (Thus the must-point-to set and must-not-point-to set are 
always empty, and the May flag is always true.) Hence, we will represent each tuple as a triple $\langle o$, unique, typestate $\rangle$.

The analysis works as follows. The first time an allocation site with an instance key $k$ is executed (during analysis), it generates the tuple $\langle k$, true, init $\rangle$. If, during the analysis, any tuple $\langle k, t r u e, s\rangle$ reaches the same (context-sensitive) allocation site, the allocation site will generate the tuple $\langle k$, false, typestate $\rangle$.

To make the above technique effective for allocation sites that are in a loop, it is necessary to find a way to "kill" the tuples where possible. This verifier utilizes a preliminary liveness analysis, computed prior to typestate checking, that determines a conservative approximation of which instance keys may be live at each program point. Whenever a tuple $p$ for an instance key $o$ flows to a program point where $o$ cannot be live, $p$ can be removed soundly.

The framework admits any form of liveness analysis, which can be plugged into the verifier. Our current implementation uses a simple bottom-up interprocedural liveness analysis, based on the results of the preliminary flow-insensitive, partially context-sensitive pointer analysis.

This approach is effective in two situations. First, singleton pattern objects clearly retain their unique predicates, and so enjoy strong updates everywhere. The Java standard libraries use singleton patterns frequently.

Additionally, the liveness analysis allows unique analysis to succeed for a ubiquitous pattern: an allocated object dies before its allocation site executes again. In practice, we have found that a simple liveness analysis catches many of these cases.

For tuples not marked unique, this verifier degenerates into the Base verifier of Sec. 3.3. For example, while uniqueness handles the handshake socket in the running example, uniqueness cannot show that the Sockets in the collection are used correctly. The instance key that represents all the Socket objects in the sockets collection is, naturally, not unique. Therefore, when the statement s.connect ( ) is analyzed, the typestate of the abstract Socket object is weakly-updated, indicating that a socket may occupy the conn state or the init state. These tuples propagate to the statement s.getOutput Stream () in talk (), causing the verifier to imprecisely report a possible error.

Note that in the example, although verifying usage of the handshake object does not rule out errors at any potential points of failure, the staged verifier will remove pairs involving the handshake object from the running verification scope. This would reduce the computational workload for the next stage.

\section{INTEGRATED TYPESTATE AND ALIAS ANALYSIS}

In this section, we describe two verifiers that make use of the access-path sets in the tuple representation. We first describe the APFocus verifier, our most precise analysis.

\subsection{Update Functions}

The interpretation of an allocation statement " $\mathrm{v}=$ new $\mathrm{T}()$ " with instance key $o$ will generate a tuple $\langle o$, true, init, $\{v\}$, false, $\emptyset\rangle$ representing the newly allocated object. When May is false, the $A P_{\text {mustNot }}$ component is redundant and, hence, initialized to be empty. Table 1 shows how a tuple is transformed by the interpretation of various statements. When a typestate method is invoked, we can (1) use the $A P_{\text {must Not }}$ information to avoid changing the typestate of the tuple where possible, (2) use the $A P_{\text {must }}$ information to perform strong updates on the tuple where possible, and (3) use the uniqueness information also to perform strong updates where possible.
When a tuple reaches the allocation site that created it, we generate two tuples, one representing the newly created object, and one representing the incoming tuple. We change the uniqueness flag to false for reasons explained earlier. For assignment statements, we update the $A P_{\text {must }}$ and $A P_{\text {mustNot }}$ as appropriate.

\subsection{Focus Operation}

We now describe the focus operation, which improves the precision of the analysis. As a motivating example, consider the statement s.connect () in the loop in the method example () in our running example. We have an incoming tuple representing all of the sockets in the collection, and, hence, we cannot apply a strong update to the tuple, which can subsequently cause a false positive. The focus operation replaces the single tuple with two tuples, one representing the object that $s$ points to, and another tuple to represent the remaining sockets. Formally, consider an incoming tuple $\left\langle o\right.$, unique, typestate, $A P_{\text {must }}$, true, $\left.A P_{\text {mustNot }}\right\rangle$ at an observable operation $e . o p()$, where $e \notin A P_{\text {must }}$, but $e$ may point to $o$ (according to the flow-insensitive points-to solution). The analysis replaces this tuple by the following two tuples:

$$
\begin{aligned}
& \left\langle o, \text { unique, typestate, } A P_{\text {must }} \cup\{e\}, \text { true, AP } P_{\text {must Not }}\right\rangle \\
& \left\langle o, \text { unique, typestate, } A P_{\text {must }}, \text { true, } A P_{\text {must Not }} \cup\{e\}\right\rangle
\end{aligned}
$$

In the example under consideration, the statement $\mathrm{s}$. connect () is reached by the tuple $\left\langle o_{1 . .5}\right.$, false, init, $\emptyset$, true, $\left.\emptyset\right\rangle$. Focusing replaces this tuple by the following two tuples:

$$
\begin{aligned}
& \left\langle o_{1 . .5}, \text { false, init, }\{s\}, \text { true }, \emptyset\right\rangle \\
& \left\langle o_{1 . .5}, \text { false, init }, \emptyset, \text { true },\{s\}\right\rangle
\end{aligned}
$$

The invocation of connect ( ) is analyzed after the focusing. This allows for a strong update on the first tuple and no update on the second tuple resulting in the two tuples:

$$
\begin{aligned}
& \left\langle o_{1 . .5}, \text { false, conn, }\{s\}, \text { true, } \emptyset\right\rangle \\
& \left\langle o_{1 . .5}, \text { false, init, } \emptyset, \text { true, }\{s\}\right\rangle
\end{aligned}
$$

We remind the reader that the unique component tuple merely indicates if multiple objects allocated at the allocation site $o$ may be simultaneously alive. A tuple such as

$\left\langle o_{1 . .5}\right.$, false, conn, $\{s\}$, true, $\left.\emptyset\right\rangle$, however, represents a single object at this point, namely the object pointed to by $s$, which allows us to use a strong update.

The analysis applies this focus operation whenever it would otherwise perform a weak update for a typestate transition. Thus, focus splits the dataflow facts tracking the two typestates that normally result from a weak update.

\subsection{Focus and polymorphism}

Polymorphism is the distinguishing feature of object-oriented languages; an object's behavior depends on its concrete type rather than it's declared type. Polymorphic call sites, present an interesting and widespread difficulty for the integrated typestate checking.

Consider the following snippet of code:

$$
\begin{aligned}
& \text { Collection } c=\ldots \\
& \text { for (Iterator } i \vec{t}=\text {. iterator }() \text {; } \\
& \quad \text { it.hasNext }() ;)\{ \\
& \text { it.next }() ;\}
\end{aligned}
$$

The Java Collections API often returns one of two Iterator implementations, depending on whether the collection is empty. Thus, the calls to both hasNext and next are polymorphic. This effectively introduces a path-sensitivity issue, where the two dynamic dispatch sites play the role of correlated branches in traditional path-sensitive discussions. 


\begin{tabular}{|c|c|}
\hline Stmt $\mathbf{S}$ & Resulting abstract tuples \\
\hline $\begin{array}{l}\text { observable operation } e . o p() \\
\text { as op } \in \Sigma \text { where } o \in p t(e)\end{array}$ & 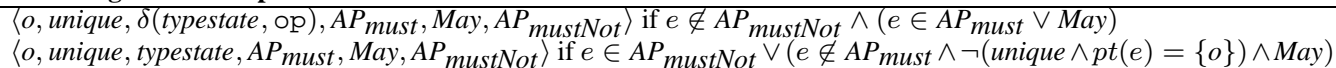 \\
\hline$v=$ new $\mathrm{T}()$ where $o=\operatorname{Stmt~} \mathrm{S}$ & $\begin{array}{l}\left\langle o, \text { false, typestate, } A P_{\text {must }} \backslash\{v \cdot \gamma \mid \gamma \in \Gamma\}, \text { May, } A P_{\text {mustNot }} \cup\{v\}\right\rangle \\
\langle o, \text { false, init },\{v\}, \text { false } \emptyset\}\rangle\end{array}$ \\
\hline$v=$ null & 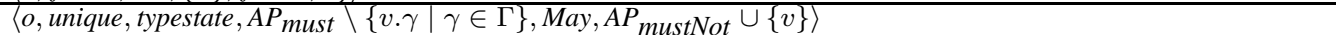 \\
\hline$v \cdot f=$ null & \begin{tabular}{ll|l}
$\left\langle o\right.$, unique, typestate,$A P_{\text {must }} \backslash\left\{e^{\prime} \cdot f \cdot \gamma\right.$ & mayAlias $\left.\left(e^{\prime}, v\right), \gamma \in \Gamma\right\}$, May,$\left.A P_{\text {must Not }} \cup\{v \cdot f\}\right\rangle$ \\
\end{tabular} \\
\hline$v=e$ & \begin{tabular}{|l|l|l}
$\left\langle o\right.$, unique, typestate,$A P_{\text {must }} \cup\{v \cdot \gamma$ & $\left.e \cdot \gamma \in A P_{\text {must }}\right\}$, May,$A P_{\text {must }}$ Not \\
$\left\langle\left\{v \mid e \in A P_{\text {must Not }}\right\}\right\rangle$
\end{tabular} \\
\hline$v \cdot f=e$ & $\begin{array}{l}A P_{\text {must }}^{\prime}:=A P_{\text {must }} \cup\left\{v \cdot f \cdot \gamma \mid e \cdot \gamma \in A P_{\text {must }}\right\} \\
\left\langle o, \text { unique, typestate }, A P_{\text {must }}^{\prime}, \text { May } \vee \exists v \cdot f \cdot \gamma \in A P_{\text {must }}^{\prime}, p \in A P\right| \text { mayAlias }(v, p) \wedge p \cdot f \cdot \gamma \notin A P_{\text {must }}^{\prime}, A P_{\text {mustNot }} \backslash \\
\left.\left\{v . f \mid e \in A P_{\text {mustNot }}\right\}\right\rangle\end{array}$ \\
\hline
\end{tabular}

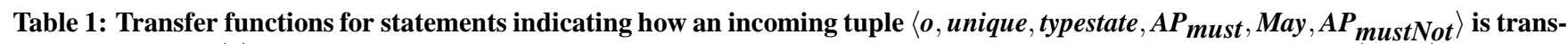
formed, where $p t(e)$ is the set of instance keys pointed-to by $e$ in the flow-insensitive solution, $v \in \operatorname{VarId}$. mayAlias $\left(e_{1}, e_{2}\right)$ iff pointer analysis indicates $e_{1}$ and $e_{2}$ may point to the same instance key.

As in ESP [11], we could introduce path-sensitive predicates that encode the direction of dynamic dispatch. Instead, our focus algorithms exploit information from the tuple to avoid propagation at polymorphic call sites.

In particular, before the call to hasNext, if we have the tuple $\langle o$, false, init, $\emptyset$, true, $\emptyset\rangle$ (in which case $o$ represents one of the two possible concrete Iterator implementations) then the focus operation will result in two tuples after the call to hasNext:

$$
\begin{aligned}
& t_{1}=\langle o, \text { false, } \text { hasNext },\{\text { it }\}, \text { true }, \emptyset\rangle \\
& t_{2}=\langle o, \text { false, } \text { init }, \emptyset, \text { true },\{\text { it }\}\rangle
\end{aligned}
$$

The flow functions for call edges exploit alias information to avoid propagating tuples down infeasible paths. In particular, the flow function for the call to it. next will not propagate $t_{2}$ to the next operation, since $t_{2}$ indicates that it must-not alias $o$. Thus, focus avoids a spurious transition to $\mathrm{err}$.

Intuitively, focus introduces a notion of path-sensitivity, where a path corresponds to a dynamic dispatch governed by alias relationships for tracked objects.

\subsection{Discarding Access Paths}

As explained earlier, we enforce limits on the length and the number of access paths allowed in the $A P_{\text {must }}$ and $A P_{\text {mustNot }}$ components to keep the number of tuples generated finite. We designed the abstract domain specifically to discard access-path information soundly, allowing heuristics that trade precision for performance but do not sacrifice soundness. This feature is crucial for scalability; the analysis would suffer an unreasonable explosion of dataflow facts if it soundly tracked every possible access path, as in much prior work [13, 23, 7, 14].

We can always safely discard access path elements from the $A P_{\text {must Not }}$ component, since the flow functions do not rely on the must-not set being complete. Additionally, we can safely discard elements from the $A P_{\text {must }}$ component by setting the May component to be true, indicating that the $A P_{\text {must }}$ set does not contain all possible aliases.

There are a variety of possible heuristic options for limiting the number of tuples. For example, ESP's "property simulation" introduced lossy joins, to merge tuples that do not differ in the typestate property of interest [11].

Our current implementation uses a different heuristic. It discards the prior $A P_{\text {mustNot }}$ paths when applying a focus operation, maintaining the more precise information from the most recent focus. This is based on intuition that in most cases the extra precision from focus will manifest at the next typestate change. This heuristic avoids a common exponential blowup in state due to a sequence of focus operations, and seems to perform well in practice.

\subsection{The APMust Verifier}

APMust is a simpler version of APFocus engine that makes use of the $A P_{\text {must }}$ component, but not the $A P_{\text {must Not }}$ component. Thus, the $A P_{\text {mustNot }}$ component is always an empty set in this abstraction. Since it does not use the $A P_{\text {mustNot }}$, it does not use focus either (since focus is ineffective without the $A P_{\text {mustNot }}$ ). Other aspects of this engine, such as the transfer functions, can be obtained in a straightforward way from the description of APFocus.

We include the APMust verifier for comparison in the next section, to help evaluate the contribution of the focus operation.

\section{EXPERIMENTAL RESULTS}

\subsection{Implementation}

The preliminary flow-insensitive pointer analysis provides a mostly context-insensitive field-sensitive Andersen's analysis [3], enhanced with a selective object sensitivity policy [26] to disambiguate contents of Java collection classes and I/O stream containers. The pointer analysis relies on an SSA register-transfer language representation of each method, which gives a measure of flow-sensitivity for points-to sets of local variables [20]. The pointer analysis names each context-sensitive allocation site as an instance key, and builds the call graph on-the-fly. For these experiments, we configure the analysis to ignore some system libraries such as java. awt and javax.swing, which generally do not have side effects that affect the typestate properties of interest. This choice reduces the computed call graph sizes.

The analysis deals with reflection by tracking objects to casts, as in $[17,25]$. When an object is created by a reflective call (e.g. newInstance), the analysis assumes (unsoundly) that the object will be cast to a declared type before being accessed. The analysis tracks these flows, and infers the type of object created by newInstance based on the declared type of relevant casts. While technically unsound, we believe that this approximation is accurate for the vast majority of reflective factory methods in Java programs.

The pointer analysis adds one-level of call-string context to calls to various library factory methods, arraycopy, and clone statements, which tend to badly pollute pointer flow precision if handled without context-sensitivity. The system uses a substantial library of models of native code behavior for the standard libraries.

The flow-sensitive combined typestate and alias analysis builds on a general Reps-Horwitz-Sagiv (RHS) IFDS tabulation solver implementation [29]. We have enhanced the standard IFDS solver in straightforward ways to handle Java's exceptional control-flow and polymorphic dispatch without undue precision loss. 


\begin{tabular}{|l||r|r|r|r|}
\hline Benchmark & Classes & Methods & Bytecode Stmts & Contexts \\
\hline bcel & 751 & 4070 & 236,271 & 6011 \\
gj & 209 & 2253 & 131,288 & 2358 \\
javacup & 102 & 567 & 45,510 & 813 \\
jbidwatcher & 492 & 2723 & 180,492 & 3641 \\
jlex & 90 & 369 & 38,019 & 610 \\
jpat-p & 39 & 115 & 10,910 & 133 \\
12j & 583 & 3443 & 209,184 & 4766 \\
lucene & 719 & 3540 & 224,478 & 5238 \\
portecle & 623 & 2992 & 210,543 & 4762 \\
rhino-a & 169 & 1150 & 81,388 & 1427 \\
sablecc-j & 362 & 2027 & 88,982 & 2476 \\
schroeder-m & 104 & 481 & 25,020 & 696 \\
soot-c & 651 & 2682 & 137,537 & 3105 \\
specjvm98 & 627 & 3465 & 290,272 & 5654 \\
symjpack-t & 52 & 204 & 73,826 & 224 \\
toba-s & 132 & 610 & 52,985 & 838 \\
tvla & 331 & 1992 & 132,422 & 9331 \\
\hline total & $\mathbf{6 0 3 6}$ & $\mathbf{3 2 , 6 8 3}$ & $\mathbf{2 , 1 6 9 , 1 2 7}$ & $\mathbf{5 2 , 0 8 3}$ \\
\hline
\end{tabular}

Table 2: Call graph characteristics for benchmarks.

\subsection{Sparsification}

To make the analysis scale, we rely on a lightweight sparsification[28] optimization prior to solving the IFDS problem. Consider an integrated verifier using access-paths bounded by depth $k$. We first consult the flow-insensitive points-to graph to conservatively determine all program variables that may appear in access-paths of depth at most $k$, which point to typestate objects of interest for a given property. Next, we perform a context-insensitive mod-ref analysis over the call graph, to determine those call graph nodes which may write to such variables; call these the relevant nodes. We prune the call graph to include only those nodes from which some relevant node is reachable, since the other nodes cannot modify the IFDS solution.

This pruning is particular important for the LocalFocus verifier. Exploiting the pruning, the LocalFocus verifier can avoid making conservative assumptions for every method call, thus greatly increasing its precision.

We assume that methods from the standard libraries will not directly transition to err, and apply sparsification accordingly. Of course, the analysis still must analyze all relevant library code to account for typestate transitions to non-err states, and aliases induced by the libraries.

In the staged verifier, we exploit results from early stages to improve sparsification in latter stages in two ways. First, if an early stage verifies that a particular statement does not transition to err, latter stages incorporate this information to improve sparsification. Second, if an early stage proves that a particular abstract object never causes an error, latter stages ignore tuples for that abstract object entirely.

\subsection{Benchmarks}

Table 2 lists the benchmarks employed in this study. Apache Bcel is a bytecode toolkit with a sample verifier. Java_cup and JLex are a parser generator and lexical analyzer, respectively, for Java. Jbidwatcher is an online auction tool. L2 $j$ is Multi-User Dungeon game server. Apache lucence is a text search engine. Portecle is a GUI application for managing secure keys and certificates. SPEC jVm98 is a collection of client-oriented applications. TVLA is a research vehicle for abstract interpretation. The

\begin{tabular}{|l|l|}
\hline Name & Description \\
\hline Enumeration & Call hasNextElement before nextElement \\
InputStream & Do not read from a closed Input St ream \\
Iterator & Do not call next without first checking hasNext \\
KeyStore & Always initialize a KeyStore before using it \\
PrintStream & Do not use a closed Print Stream \\
PrintWriter & Do not use a closed PrintWriter \\
Signature & Follow initialization phases for Signatures \\
Socket & Do not use a Socket until it is connected \\
Stack & Do not peek or pop an empty Stack \\
URLConn & Illegal operation performed when already connected \\
Vector & Do not access elements of an empty Vector \\
\hline
\end{tabular}

Table 3: Typestate properties.

remainder of the benchmarks come from the Ashes suite, described at the Ashes web page ${ }^{3}$.

The Table reports size characteristics restricted to methods discovered by on-the-fly call graph construction. The call graph includes methods from both the application and the libraries; for many programs the size of the program analyzed is dominated by the standard libraries. The table also reports the number of (method) contexts in the call graph. Recall that the context-sensitivity policy models some methods with multiple contexts.

Table 3 lists intuitive descriptions of the typestate properties verified in the experiments.

\subsection{Methodology}

The experiments evaluate the following verification algorithms:

- FI: flow-insensitive analysis (Sec. 2.2.1)

- LocalFocus: the intraprocedural analysis (Sec. 2.2.2)

- Base: the base analysis (Sec. 3.3)

- Unique: the analysis using the unique reasoning (Sec. 4)

- APMust: the integrated analysis without focus (Sec. 5)

- APFocus: the integrated analysis with focus (Sec. 5.)

- Staged: a staged analysis consisting of three stages: LocalFocus, Unique, and APFocus.

Note that each verifier performs the FI analysis as a first step, since it is extremely fast and can prune the workload based on the "verification scope" passed from the previous stage. The experiments use an access-path depth limit of 2, and unlimited access-path set width.

All experiments ran on an IBM Intellistation Z pro with two 3.06 $\mathrm{GHz}$ Intel Xeon CPUs and 3.62 GB of RAM, running Windows XP. The analysis implementation, consisting of roughly 200,000 lines of Java code, ran on the IBM J2RE 1.4.2 for Windows, with a max heap of $800 \mathrm{MB}$.

\subsection{Results}

Figure 4 shows the percentage of warnings, as a percentage of total number of statements that the callgraph indicates might transition to $\operatorname{err}$ (points of potential failure (PPF)). The number shown above each bar in the figure is the total number of PPFs.

The rightmost cluster of bars shows the total number of warnings across all runs. Overall,

- The FI verifier verifies correctness for $\mathbf{3 0 \%}$ of PPFs.

${ }^{3}$ http://www.sable.mcgill.ca/ashes/ 

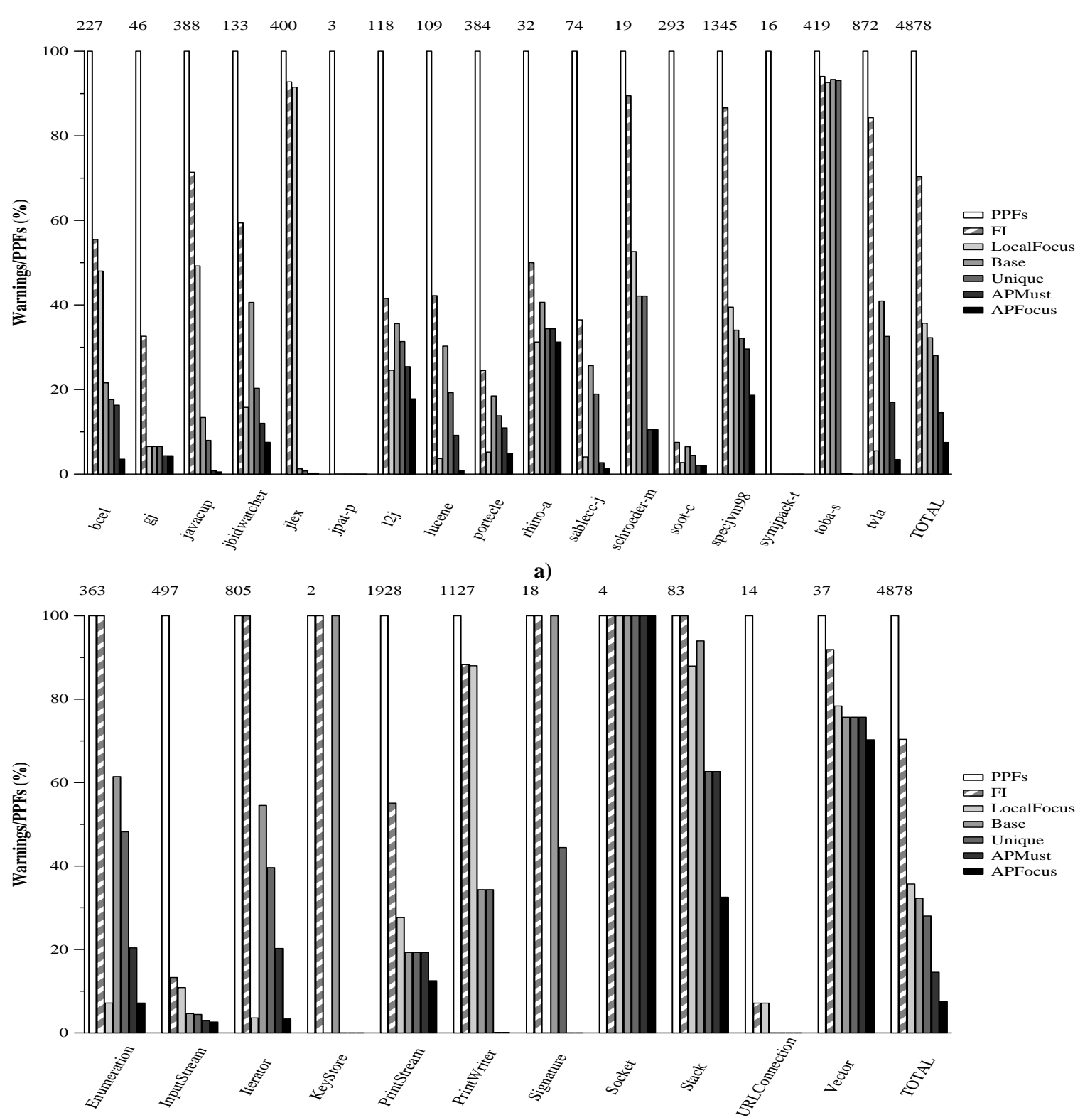

b)

Figure 4: Percentage of warnings out of total number of points of potential failure (PPFs). Results are grouped by a) application, and b) property. Number of PPFs is shown above each group.

- The LocalFocus verifier verifies correctness for $\mathbf{6 4 \%}$ of PPFs.

- The Base verifier verifies correctness for $\mathbf{6 8 \%}$ of PPFs.

- The Unique verifier verifies correctness for $\mathbf{7 2 \%}$ of PPFs.

- The APMust verifier verifies correctness for $\mathbf{8 5 \%}$ of PPFs.

- The APFocus verifier verifies correctness for $\mathbf{9 3 \%}$ of PPFs.

Table 4 shows detailed results for verification warnings produced by the most precise (APFocus) solver. By construction, the Staged verifier has the same precision as APFocus. Sec. 6.7 discusses the sources of many false positives.

\subsection{Performance}

Figure 5 reports the running times of the various verifiers across the benchmarks. The results show the expected relative costs of the various verifiers.

\subsubsection{Impact of Staging}

The Staged verifier improves performance compared to the APFocus verifier on 9 of the 10 codes where typestate checking takes more than 30 seconds. On these 10 codes, staging improves performance by up to $85 \%$ (tvla), with a median of $34 \%$. Staging hurts performance by $40 \%$ on $12 j$; on this code, many PPFs survive early verification stages, and the cost/precision tradeoffs of the various solvers do not pay off. 


\begin{tabular}{|c|c|c|c|c|c|c|c|c|c|c|c|c|c|}
\hline & Enum & InptStr & Itr & KStore & PrntStr & PrntWr & Sig & Socket & Stack & URLConn & Vector & \multicolumn{2}{|c|}{ Total } \\
\hline bcel & $0 / 2$ & $0 / 1$ & $0 / 15$ & & $0 / 36$ & $0 / 139$ & & & $8 / 32$ & & $0 / 2$ & $8 / 227$ & $3.5 \%$ \\
\hline $\mathrm{gj}$ & & $2 / 6$ & & & $0 / 40$ & & & & & & & $2 / 46$ & $4.4 \%$ \\
\hline javacup & $0 / 82$ & $0 / 6$ & & & $0 / 111$ & $0 / 166$ & & & $2 / 23$ & & & $2 / 388$ & $0.5 \%$ \\
\hline jbidwatcher & $1 / 8$ & $0 / 9$ & $0 / 46$ & & $0 / 31$ & $0 / 9$ & & & & $0 / 13$ & $9 / 17$ & $10 / 133$ & $7.5 \%$ \\
\hline jlex & $0 / 5$ & & & & $0 / 29$ & $0 / 365$ & & & $1 / 1$ & & & $1 / 400$ & $0.3 \%$ \\
\hline jpat-p & & & & & $0 / 3$ & & & & & & & $0 / 3$ & $0.0 \%$ \\
\hline $12 j$ & & $6 / 36$ & $0 / 17$ & & $0 / 48$ & & & $4 / 4$ & $1 / 3$ & & $10 / 10$ & $21 / 118$ & $17.8 \%$ \\
\hline lucene & $0 / 29$ & $0 / 6$ & $0 / 11$ & & $0 / 60$ & $0 / 1$ & & & & & $1 / 2$ & $1 / 109$ & $0.9 \%$ \\
\hline portecle & $19 / 72$ & $0 / 266$ & $0 / 1$ & $0 / 2$ & $0 / 25$ & & $0 / 18$ & & & & & $19 / 384$ & $5.0 \%$ \\
\hline rhino-a & $3 / 9$ & & & & $0 / 16$ & $1 / 1$ & & & $6 / 6$ & & & $10 / 32$ & $31.3 \%$ \\
\hline sablecc-j & $0 / 24$ & & & & $0 / 47$ & & & & $1 / 3$ & & & $1 / 74$ & $1.4 \%$ \\
\hline schroeder-m & $0 / 6$ & $2 / 11$ & & & $0 / 2$ & & & & & & & $2 / 19$ & $10.5 \%$ \\
\hline Soot-c & $0 / 14$ & $0 / 2$ & & & $0 / 213$ & $0 / 58$ & & & & & $6 / 6$ & $6 / 293$ & $2.0 \%$ \\
\hline specjvm98 & $3 / 109$ & $3 / 151$ & & & $241 / 1075$ & & & & $4 / 9$ & $0 / 1$ & & $251 / 1345$ & $18.7 \%$ \\
\hline symjpack-t & & & & & $0 / 16$ & & & & & & & $0 / 16$ & $0.0 \%$ \\
\hline toba-s & $0 / 3$ & $0 / 3$ & & & $0 / 25$ & $0 / 386$ & & & $1 / 2$ & & & $1 / 419$ & $0.2 \%$ \\
\hline tvla & & & $27 / 715$ & & $0 / 151$ & $0 / 2$ & & & $3 / 4$ & & & $30 / 872$ & $3.4 \%$ \\
\hline Total & $\begin{array}{r}26 / 363 \\
7.2 \%\end{array}$ & $\begin{array}{r}13 / 497 \\
2.6 \%\end{array}$ & $\begin{array}{r}27 / 805 \\
3.4 \%\end{array}$ & $\begin{array}{l}0 / 2 \\
0.0 \%\end{array}$ & $\begin{array}{r}241 / 1928 \\
12.5 \%\end{array}$ & $\begin{array}{r}1 / 1127 \\
0.1 \%\end{array}$ & $\begin{array}{l}0 / 18 \\
0.0 \%\end{array}$ & $\begin{array}{r}4 / 4 \\
100.0 \%\end{array}$ & $\begin{array}{l}27 / 83 \\
32.5 \%\end{array}$ & $\begin{array}{l}0 / 14 \\
0.0 \%\end{array}$ & $\begin{array}{l}26 / 37 \\
70.3 \%\end{array}$ & $365 / 4878$ & $7.5 \%$ \\
\hline
\end{tabular}

Table 4: Findings for the most precise (staged) solver across all benchmarks and typestate properties. Each entry in the table shows the number of warnings as a fraction of the number of PPFs, for each benchmark/property combination.

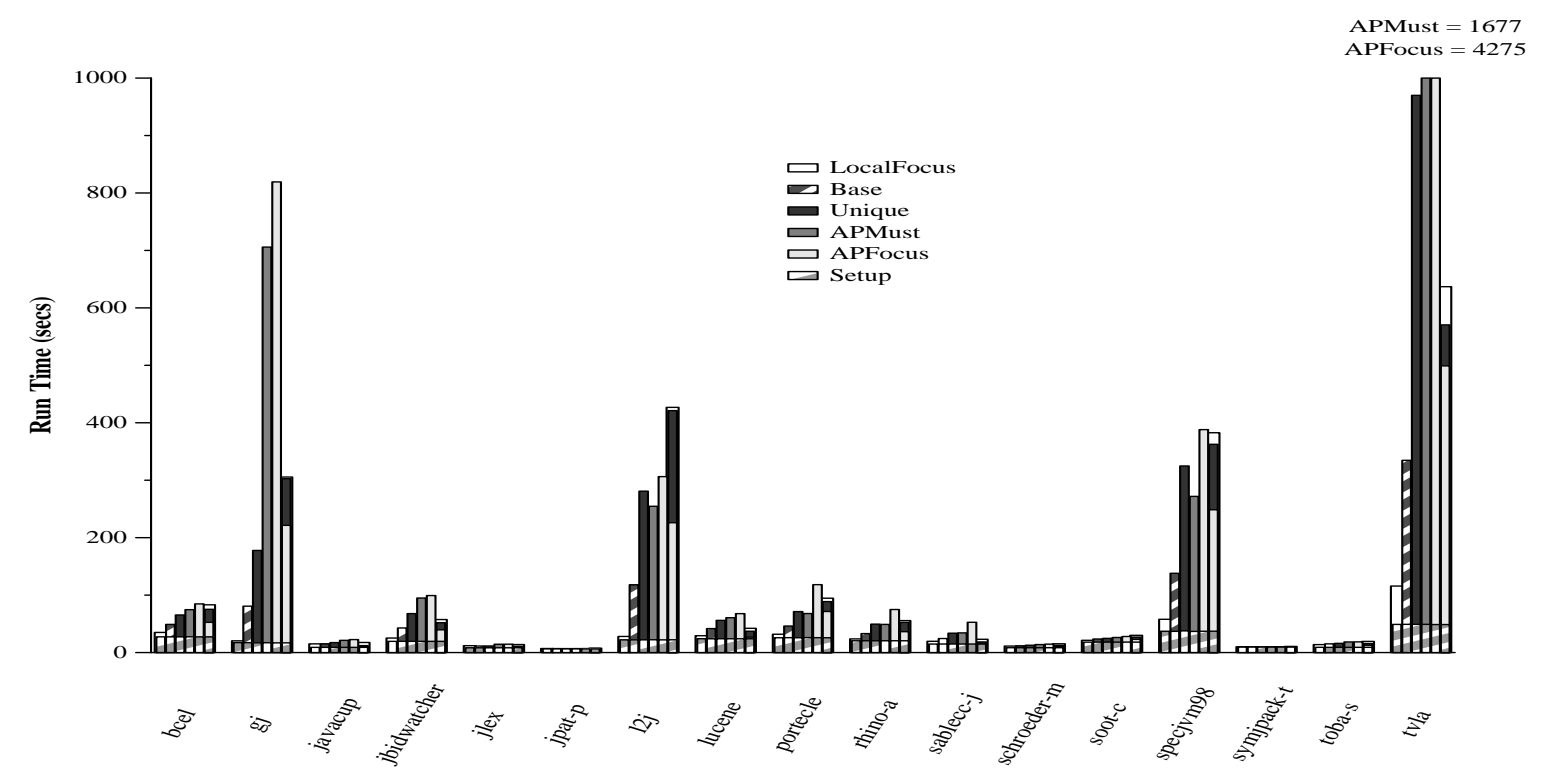

Figure 5: Total wallclock time needed to run the analysis. "Setup" indicates the preliminary activities; primarily the preceding flow-insensitive pointer analysis and call graph construction. The rightmost stacked bar in each group represents the running time of the Staged verifier.

\subsubsection{Impact of Sparsification}

We evaluated the sparsification of Sec. 6.2 across all runs of the staged verifier. With sparsification, $70 \%$ of supergraphs have fewer than 3500 nodes, $95 \%$ have fewer than 25,000 nodes, and $100 \%$ have fewer than 40,000 nodes. The corresponding numbers without sparsification are drastically higher: roughly $80 \%$ of unpruned supergraphs have more than 125,000 nodes, and $20 \%$ have over 290,000 nodes. Overall, sparsification reduces median supergraph size by roughly a factor of 50 . We would expect a corresponding reduction in space and running time, if we could run the unpruned verifiers without running out of memory.

\subsubsection{Impact of Initial Pointer Analysis}

The precision of the preceding flow-insensitive pointer analysis significantly impact on performance and precision. A more accu- rate pointer analysis allows better sparsification, more effective live analysis and improved disambiguation overall. We ran many of the analyses with a context-insensitive Andersen-style pointer analysis, without the custom context-sensitivity policies described earlier. Many benchmarks timed out on several rules; we conclude that adequate precision in the preceding pointer analysis is vital.

Our context-sensitivity policy employs object-sensitivity for types from the standard libraries typically relevant to these typestate properties (namely collections and I/O streams). Some benchmarks defeat this object-sensitivity policy by using application-level collections or streams. For example, TVLA uses a library of applicationlevel collections, and specJVM98 uses a reporting library of custom I/O streams. To handle these cases more effectively, we need to infer a pointer-analysis context-sensitivity policy for application classes that match typestate properties. Iterative refinement techniques $[27,19]$ may apply to this problem. 


\subsection{Discussion}

Overall, the results show that our combination of techniques is relatively successful and efficient at verifying these typestate properties. The various techniques complement each other, contributing to the effectiveness of the staged verifier.

Since our goal in this paper is the successful verification of typestate properties, we have deliberately chosen a set of mature benchmarks. For our experiments, we assume that typestate violations in these benchmarks are all false alarms. We have examined, by hand, many of the warnings which our most precise verifier does not eliminate.

The specJVM98 code's use of PrintStream accounts for 241 of the 365 warnings reported. These are all false positives, stemming from a few lines of code in the specJVM98 harness. This program stores a PrintStream object in a static field Context.out, and uses the object ubiquitously throughout the various benchmarks. The particular idiom by which the program caches the PrintStream object in a static field defeats our focus heuristics, leading to a loss of precision.

Of the remaining 124 warnings, 53 arise from the Vector and Stack properties. Most of these warnings appear to represent a failure of the typestate property to capture all legal behavior, as opposed to solver limitations. For example, our typestate property for Vector does not account for the return value from Vector.size(). Many times, application code accesses a Vector via statements guarded by a test that size $>0$. This pattern accounts for many of the false positives for the Stack and Vector rules. For proper treatment, these APIs require at least range-check analysis, as commonly applied to array-bounds checking (e.g. [18]).

The remaining warnings appear to arise from a combination of analysis approximations and typestate property limitations.

We expect that in the near future we can improve precision by a) access-path tracking for objects that are not typestate objects, but are likely to point to them, and b) increasing the scope of focus by exploiting inexpensive local alias reasoning. We suspect that substantial improvements in alias precision are within reach, without undue performance compromise.

In many cases, programmers deduce from application logic that a particular iterator must have a next element, or a particular collection must not be empty. The typestate property for a single object does not allow for application logic which ensures, via some back door, that an object occupies a particular typestate. Designing efficient, effective analysis for more general specifications remains a difficult problem.

\section{RELATED WORK}

Many existing verification frameworks (e.g., $[11,4,8]$ ) use a two-phased approach, performing points-to analysis as a preceding phase, followed by typestate checking. This approach only supports weak updates as discussed in Sec. 3.3.

The current version of ESP [13] uses an integrated approach, recording must and may alias information in a flow-sensitive manner. They observe that the may set becomes polluted and expensive to maintain, and even hint toward maintaining a must-not set as a possible future solution. In contrast, our approach adds must-not and also introduces the notions of uniqueness and focus, and uses staging to achieve increased scalability and precision.

DeLine and Fähndrich [12] present a type system for typestate properties for objects. Their system guarantees that a program that typechecks has no typestate violations, and provides a modular, sound checker for object-oriented programs. To handle aliasing, they employ the adoption and focus operations to a linear type sys- tem, as described in [15]. With these operations, the type checker can assume must-alias properties for a limited program scope, and thus apply strong updates allowing typestate transitions. Our approach can prove correctness of a more general class of programs, since a context-sensitive analysis can accept programs for which an expression cannot be assigned a unique type at a given program point. Furthermore, our focus operation generates facts that can flow across arbitrary program scopes, in contrast to the limited program scope handled by [15]. On the other hand, our approach is non-modular and thus more expensive.

Aiken et al. [1] present an inference algorithm for inferring restricted and confined pointers, which they use to enable strong updates. We believe that the focusing technique we exploit, inspired by [30], can sometimes achieve a similar effect without explicitly inferring restricted and confined pointers, and sometimes enable strong updates even when the pointers are not restricted/confined. Further, the uniqueness technique we use provides a somewhat orthogonal, cheap, technique for enabling strong updates.

Field et al. [16] present algorithms based on abstractions that integrate alias and typestate information, but restricted to shallow programs, with only single-level pointers to typestate objects.

The parametric shape analysis presented in [31] has served as the basis for very precise verification algorithms, where the verification is integrated with heap analysis (e.g., [35].) These algorithms, however, do not scale well. We plan to extend our staged verifier by adding such precise verifiers as a last stage.

Counter-example guided refinement $[5,22]$ based approaches have had impressive results in certain domains. But they have so far been less successful in dealing with complex heap manipulation, partly because these approaches attempt to automatically derive appropriate heap analyses. Our staged verifier has a "refinement" flavor, but restricted to a fixed set of manually crafted verifiers.

Aliasing of our combined domain resembles previous approaches to flow-sensitive, context-sensitive access-path-based pointer analysis [23, 7]. Emami, Ghiya and Hendren [14] presented a domain that combined may and must points-to information. Our IFDSbased solvers memoize function summaries, similar to Wilson and Lam's partial transfer functions [34]. Our domain differs from these previous works since a) it tracks must and must-not paths, but not may, and b) Java's strong typing avoids complications arising from pointers to stack locations.

Iterative refinement techniques [27, 19] perform pointer analysis in multiple passes, with a client-independent first pass, followed by subsequent passes using context-sensitivity policies driven by client feedback. In future work we plan to integrate these techniques into our framework, where each typestate solver provides feedback for the next stage's pointer analysis.

\section{REFERENCES}

[1] A. Aiken, J. S. Foster, J. Kodumal, and T. Terauchi. Checking and inferring local non-aliasing. ACM SIGPLAN Notices, 38(5):129-140, May 2003. In Conference on Programming Language Design and Implementation (PLDI).

[2] R. Alur, P. Cerny, P. Madhusudan, and W. Nam. Synthesis of interface specifications for java classes. SIGPLAN Not., 40(1):98-109, 2005.

[3] L. O. Andersen. Program Analysis and Specialization for the C Programming Language. PhD thesis, DIKU, Univ. of Copenhagen, May 1994. (DIKU report 94/19).

[4] T. Ball, R. Majumdar, T. Millstein, and S. Rajamani. Automatic predicate abstraction of C programs. In Proc. ACM Conf. on Programming Language Design and Implementation, pages 203-213, June 2001. 
[5] T. Ball and S. K. Rajamani. The SLAM project: debugging system software via static analysis. ACM SIGPLAN Notices, 37(1):1-3, Jan. 2002.

[6] D. R. Chase, M. Wegman, and F. K. Zadeck. Analysis of pointers and structures. ACM SIGPLAN Notices, 25(6):296-310, June 1990. In PLDI.

[7] J.-D. Choi, M. Burke, and P. Carini. Efficient flow-sensitive interprocedural computation of pointer-induced aliases and side effects. In POPL 93, pages 232-245, 1993.

[8] J. Corbett, M. Dwyer, J. Hatcliff, C. Pasareanu, Robby, S. Laubach, and H. Zheng. Bandera: Extracting finite-state models from Java source code. In Proc. Intl. Conf. on Software Eng., pages 439-448, June 2000.

[9] P. Cousot and R. Cousot. Systematic design of program analysis frameworks. In Proc. ACM Symp. on Principles of Programming Languages, pages 269-282, New York, NY, 1979. ACM Press.

[10] M. Das. Unification-based pointer analysis with directional assignments. ACM SIGPLAN Notices, 35(5):35-46, May 2000. In Conference on Programming Language Design and Implementation (PLDI).

[11] M. Das, S. Lerner, and M. Seigle. ESP: path-sensitive program verification in polynomial time. ACM SIGPLAN Notices, 37(5):57-68, May 2002. In Conference on Programming Language Design and Implementation (PLDI).

[12] R. DeLine and M. Fähndrich. Typestates for objects. In 18th European Conference on Object-Oriented Programming (ECOOP), volume 3086 of LNCS, June 2004.

[13] N. Dor, S. Adams, M. Das, and Z. Yang. Software validation via scalable path-sensitive value flow analysis. In ISSTA, pages 12-22, 2004.

[14] M. Emami, R. Ghiya, and L. J. Hendren. Context-sensitive interprocedural points-to analysis in the presence of function pointers. ACM SIGPLAN Notices, 29(6):242-256, June 1994. In Conference on Programming Language Design and Implementation (PLDI).

[15] M. Fahndrich and R. DeLine. Adoption and focus: practical linear types for imperative programming. ACM SIGPLAN Notices, 37(5):13-24, May 2002. In Conference on Programming Language Design and Implementation (PLDI).

[16] J. Field, D. Goyal, G. Ramalingam, and E. Yahav. Typestate verification: Abstraction techniques and complexity results. In Proc. of Static Analysis Symposium (SAS'03), volume 2694 of $L N C S$, pages 439-462. Springer, June 2003.

[17] S. Fink, J. Dolby, and L. Colby. Semi-automatic J2EE transaction configuration. Technical Report RC23326, IBM, 2004.

[18] R. Gupta. Optimizing array bound checks using flow analysis. ACM Lett. Program. Lang. Syst., 2(1-4):135-150, 1993.

[19] S. Guyer and C. Lin. Client-driven pointer analysis. In Proc. of SAS'03, volume 2694 of $L N C S$, pages 214-236, June 2003.

[20] R. Hasti and S. Horwitz. Using static single assignment form to improve flow-insensitive pointer analysis. ACM SIGPLAN Notices, 33(5):97-105, May 1998. In Conference on Programming Language Design and Implementation (PLDI).

[21] N. Heintze and O. Tardieu. Ultra-fast aliasing analysis using CLA: A million lines of C code in a second. ACM SIGPLAN Notices, 36(5):254-263, May 2001. In Conference on Programming Language Design and Implementation (PLDI).
[22] T. A. Henzinger, R. Jhala, R. Majumdar, and G. Sutre. Lazy abstraction. In Symposium on Principles of Programming Languages, pages 58-70, 2002.

[23] W. Landi and B. G. Ryder. A safe approximate algorithm for interprocedural aliasing. ACM SIGPLAN Notices, 27(7):235-248, July 1992. In Conference on Programming Language Design and Implementation (PLDI).

[24] O. Lhoták and L. Hendren. Scaling Java points-to analysis using SPARK. In 12th International Conference on Compiler Construction (CC), volume 2622 of $L N C S$, pages 153-169, Apr. 2003.

[25] B. Livshits, J. Whaley, and M. S. Lam. Reflection analysis for java. In Proceedings of Programming Languages and Systems: Third Asian Symposium, APLAS 2005, November 2005.

[26] A. Milanova, A. Rountev, and B. G. Ryder. Parameterized object sensitivity for points-to analysis for java. ACM Trans. Softw. Eng. Methodol., 14(1):1-41, 2005.

[27] J. Plevyak and A. A. Chien. Precise concrete type inference for object-oriented languages. ACM SIGPLAN Notices, 29(10):324-324, Oct. 1994. In Conference on Object-Oriented Programming Systems, Languages, and Applications (OOPSLA).

[28] G. Ramalingam. On sparse evaluation representations. Theor. Comput. Sci., 277(1-2):119-147, 2002.

[29] T. Reps, S. Horwitz, and M. Sagiv. Precise interprocedural dataflow analysis via graph reachability. In Conference record of POPL '95, 22nd ACM SIGPLAN-SIGACT Symposium on Principles of Programming Languages, pages 49-61, New York, NY, USA, 1995. ACM Press.

[30] M. Sagiv, T. Reps, and R. Wilhelm. Parametric shape analysis via 3-valued logic. In Proc. ACM Symp. on Principles of Programming Languages, pages 105-118, 1999.

[31] M. Sagiv, T. Reps, and R. Wilhelm. Parametric shape analysis via 3-valued logic. Transactions on Programming Languages and Systems (TOPLAS), 24(3):217-298, May 2002.

[32] R. E. Strom and S. Yemini. Typestate: A programming language concept for enhancing software reliability. IEEE Trans. Software Eng., 12(1):157-171, 1986.

[33] J. Whaley, M. Martin, and M. Lam. Automatic extraction of object-oriented component interfaces. In Proceedings of the International Symposium on Software Testing and Analysis, July 2002.

[34] R. P. Wilson and M. S. Lam. Efficient context-sensitive pointer analysis for $\mathrm{C}$ programs. ACM SIGPLAN Notices, 30(6):1-12, June 1995. In Conference on Programming Language Design and Implementation (PLDI).

[35] E. Yahav and G. Ramalingam. Verifying safety properties using separation and heterogeneous abstractions. In Proceedings of the ACM SIGPLAN 2004 conference on Programming language design and implementation, pages 25-34. ACM Press, 2004. 